\title{
TENSOR PRODUCTS AND THE JOINT SPECTRUM IN HILBERT SPACES
}

\author{
ZOIA CEAUŞESCU AND F.-H. VASILESCU
}

\begin{abstract}
Given two complex Hilbert spaces $X$ and $Y$ and two commuting systems of linear continuous operators $a=\left(a_{1}, \ldots, a_{n}\right)$ on $X$ and $b=$ $\left(b_{1}, \ldots, b_{m}\right)$ on $Y$, the joint spectrum of the commuting system $\left(a_{1} \otimes\right.$ $\left.1, \ldots, a_{n} \otimes 1,1 \otimes b_{1}, \ldots, 1 \otimes b_{m}\right)$ on $X \bar{\otimes} Y$ is expressed by the Cartesian product of the joint spectrum of $a$ with the joint spectrum of $b$.
\end{abstract}

1. Introduction. Let $X, Y$ be two complex Hilbert spaces. We denote by $X \bar{\otimes} Y$ the completion of the tensor product $X \otimes Y$ with respect to the canonical scalar product. Analogously, if $X_{1}, \ldots, X_{n}$ is an arbitrary finite family of complex Hilbert spaces, we denote by $X_{1} \bar{\otimes} \ldots \bar{\otimes} X_{n}$ the canonical completion of the tensor product $X_{1} \otimes \cdots \otimes X_{n}$. For every Hilbert space $X$ let $L(X)$ be the algebra of all bounded linear operators on $X$. If $a=\left(a_{1}, \ldots, a_{n}\right) \subset L(X)$ is a commuting system then we denote by $\sigma(a, X)$ the joint spectrum of $a$ on $X$ in the sense of [5].

In a previous paper [2] we have shown that if $X_{1}, \ldots, X_{n}$ are Hilbert spaces, $a_{j} \in L\left(X_{j}\right)$ are arbitrary and

$$
\tilde{a}_{j}=1 \otimes \cdots \otimes 1 \otimes a_{j} \otimes 1 \otimes \cdots \otimes 1 \in L(X) \quad(j=1, \ldots, n),
$$

where $X=X_{1} \bar{\otimes} \ldots \bar{\otimes} X_{n}$, if we define $\tilde{a}=\left(\tilde{a}_{1}, \ldots, \tilde{a}_{n}\right)$ then we have the equality

$$
\sigma(\tilde{a}, X)=\sigma\left(a_{1}, X_{1}\right) \times \cdots \times \sigma\left(a_{n}, X_{n}\right) .
$$

The formula (1.1) is (on Hilbert spaces) a refinement of a result of A. T. Dash and $\mathrm{M}$. Schechter [3].

The aim of this paper is to give, in a certain sense, the definitive form of (1.1). Namely, we replace in (1.1) the operators $a_{j}$ with commuting systems on $X_{j}(j=1, \ldots, n)$ and prove a similar result about them (see Corollary 2.3 below).

Let us recall some definitions and results concerning the joint spectrum [5], [6]. If $X$ is a fixed Hilbert space and $s=\left(s_{1}, \ldots, s_{n}\right)$ a system of indeterminates, we denote by $\Lambda[s, X]$ the tensor product $\Lambda[s] \otimes X$, where $\Lambda[s]$ is the exterior algebra over the complex field generated by $s_{1}, \ldots, s_{n}$. Analogously, $\Lambda^{p}[s, X]$ is the tensor product $\Lambda^{p}[s] \otimes X$, where $\Lambda^{p}[s]$ is the vector subspace of $\Lambda[s]$ containing all the exterior forms of degree $p(p=0,1, \ldots, n)$. An element $\xi \in \Lambda^{p}[s, X]$ will be written as

Received by the editors May 20, 1977 and, in revised form, February 21, 1978.

AMS (MOS) subject classifications (1970). Primary 47A10; Secondary 46M05, 55J25.

Key words and phrases. Tensor product, Hilbert space, joint spectrum. 


$$
\xi=\sum_{1<j_{1}<\cdots<j_{p}<n} x_{j_{1} \ldots j_{p}} s_{j_{1}} \wedge \cdots \wedge s_{j_{p}} \quad\left(x_{j_{1} \ldots j_{p}} \in X\right),
$$

hence the symbol $\otimes$ will be omitted.

The space $\Lambda^{p}[s, X]$ can be identified with a direct sum of $\left(\begin{array}{l}n \\ p\end{array}\right)$ copies of $X$, therefore $\Lambda^{p}[s, X]$ can be endowed with a natural structure of Hilbert space. We have also

$$
\Lambda[s, X]=\bigoplus_{p=0}^{n} \Lambda^{p}[s, X] .
$$

Consider now a commuting system $a=\left(a_{1}, \ldots, a_{n}\right) \subset L(X)$. Denote by $\delta_{a} \in L(\Lambda[s, X])$ the operator given by

$$
\delta_{a} \xi=\left(a_{1} s_{1}+\cdots+a_{n} s_{n}\right) \wedge \xi \quad(\xi \in \Lambda[s, X]) .
$$

We have $K\left(\delta_{a}\right) \supset R\left(\delta_{a}\right)$ (where $K$ and $R$ denote the kernel and the range, respectively). The system $a$ is said to be nonsingular (singular) on $X$ if $K\left(\delta_{a}\right)=R\left(\delta_{a}\right)\left(K\left(\delta_{a}\right) \neq R\left(\delta_{a}\right)\right)$. The set of all $z=\left(z_{1}, \ldots, z_{n}\right) \in \mathbf{C}^{n}$ with the property that $z-a$ is singular on $X$ is called the (joint) spectrum of $a$ on $X$, denoted by $\sigma(a, X)[5]$.

Finally, we use the fact that $a=\left(a_{1}, \ldots, a_{n}\right) \subset L(X)$ is nonsingular on $X$ if and only if the operator $\alpha(a)=\delta_{a}+\delta_{a}^{*}$ is invertible on $\Lambda[s, X]$ (see [6]).

2. The main result. First of all we need the following

2.1. LemMA. Let $X$ be a Hilbert space, $a=\left(a_{1}, \ldots, a_{n}\right) \subset L(X)$ a commuting system and $Y(\neq 0)$ another Hilbert space. The system $a=\left(a_{1}, \ldots, a_{n}\right)$ is nonsingular on $X$ if and only if the system $\tilde{a}=\left(a_{1} \otimes 1, \ldots, a_{n} \otimes 1\right)$ is nonsingular on $X \bar{\otimes} Y$.

Proof. The proof of this result is contained in [2]. For the convenience of the reader we give a sketch of it.

Let us fix a system of indeterminates $s=\left(s_{1}, \ldots, s_{n}\right)$. Then the spaces $\Lambda[s, X \bar{\otimes} Y]$ and $\Lambda[s, X] \bar{\otimes} Y$ can be identified in a natural way by setting

$$
u\left(x \otimes y s_{j_{1}} \wedge \cdots \wedge s_{j_{p}}\right)=\left(x s_{j_{1}} \wedge \cdots \wedge s_{j_{p}}\right) \otimes y \quad(x \in X, y \in Y)
$$

and extending $u$ as a unitary operator from $\Lambda[s, X \bar{\otimes} Y$ onto $\Lambda[s, X] \bar{\otimes} Y$. Moreover, we have $u \delta_{\tilde{a}}=\left(\delta_{a} \otimes 1\right) u$, whence

$$
\alpha(\tilde{a})=u^{*}(\alpha(a) \otimes 1) u
$$

The relation (2.1) shows that $\alpha(\tilde{a})$ is invertible if and only if $\alpha(a) \otimes 1$ is invertible, which in turn is equivalent to the invertibility of $\alpha(a)$ (see, for example [1]).

Let us suppose that $a=\left(a_{1}, \ldots, a_{n}\right)$ and $b=\left(b_{1}, \ldots, b_{m}\right)$ are two systems of operators acting on the same Hilbert space $X$. Then the system $\left(a_{1}, \ldots\right.$, $\left.a_{n}, b_{1}, \ldots, b_{m}\right)$ will be denoted by $(a, b)$.

We present now the main result of this paper. The proof is suggested by the arguments which lead to the tensor formula of Künneth [4]. 
2.2. TheOREM. Let $X$ and $Y$ be two nonnull complex Hilbert spaces and $a=\left(a_{1}, \ldots, a_{n}\right) \subset L(X), b=\left(b_{1}, \ldots, b_{m}\right) \subset L(Y)$ two commuting systems. If we define the systems $\tilde{a}=\left(a_{1} \otimes 1, \ldots, a_{n} \otimes 1\right)$ and $\tilde{b}=\left(1 \otimes b_{1}, \ldots, 1 \otimes\right.$ $\left.b_{m}\right)$ in $L(X \bar{\otimes} Y)$ then we have the relation

$$
\sigma((\tilde{a}, \tilde{b}), X \bar{\otimes} Y)=\sigma(a, X) \times \sigma(b, Y) .
$$

PRoof. According to the projection property of the joint spectrum [5] and to Lemma 2.1, we have the relations

$$
\sigma((\tilde{a}, \tilde{b}), X \bar{\otimes} Y) \subset \sigma(\tilde{a}, X \bar{\otimes} Y) \times \sigma(b, X \bar{\otimes} Y)=\sigma(a, X) \times \sigma(b, Y) .
$$

Let us prove the converse inclusion. Consider first $s=\left(s_{1}, \ldots, s_{n}\right)$ and $t=\left(t_{1}, \ldots, t_{m}\right)$ two systems of indeterminates and let $(s, t)$ be the system $\left(s_{1}, \ldots, s_{n}, t_{1}, \ldots, t_{m}\right)$. Then the spaces $\Lambda[(s, t), X \otimes Y]$ and $\Lambda[s, X] \otimes$ $\Lambda[t, Y]$ can be identified in a natural way. Indeed, let us define the map

$$
\begin{aligned}
v\left(x \otimes y s_{j_{1}} \wedge \cdots \wedge\right. & \left.s_{j_{p}} \wedge t_{k_{1}} \wedge \cdots \wedge t_{k_{q}}\right) \\
& =\left(x s_{j_{1}} \wedge \cdots \wedge s_{j_{p}}\right) \otimes\left(y t_{k_{1}} \wedge \cdots \wedge t_{k_{q}}\right)
\end{aligned}
$$

where $x \in X, y \in Y, p \in\{1, \ldots, n\}$ and $q \in\{1, \ldots, m\}$ are arbitrary. The map $v$ can be extended as a unitary operator from $\Lambda[(s, t), X \bar{\otimes} Y]$ onto $\Lambda[s, X] \otimes \Lambda[t, Y]$. This fact will follow from the equality

$$
\Lambda^{k}[(s, t), X \bar{\otimes} Y]=\underset{p+q=k}{\bigoplus^{p, q}}[(s, t), X \bar{\otimes} Y] \quad(k=0,1, \ldots, m+n),
$$

where $\Lambda^{p, q}[(s, t), X \bar{\otimes} Y]$ is the set of forms of degree $p$ in $s_{1}, \ldots, s_{n}$ and of degree $q$ in $t_{1}, \ldots, t_{m}$, and from the equality

$$
\begin{aligned}
& \left\|v\left(\sum_{\substack{j_{1}<\cdots<j_{p} \\
k_{1}<\cdots<k_{q}}} x_{j_{1} \ldots j_{p}} \otimes y_{k_{1} \ldots k_{q}} s_{j_{1}} \wedge \cdots \wedge s_{j_{p}} \wedge t_{k_{1}} \wedge \cdots \wedge t_{k_{q}}\right)\right\|^{2} \\
& \quad=\left\|\sum_{\substack{j_{1}<\cdots<j_{p} \\
k_{1}<\cdots<k_{q}}} x_{j_{1} \ldots j_{p}} \otimes y_{k_{1} \ldots k_{q}} s_{j_{1}} \wedge \cdots \wedge s_{j_{p}} \wedge t_{k_{1}} \wedge \cdots \wedge t_{k_{q}}\right\|^{2} .
\end{aligned}
$$

By a straightforward calculation we obtain that

$$
v \delta_{(\tilde{a}, \tilde{b})}=\left(\delta_{a} \otimes 1+\zeta \otimes \delta_{b}\right) v,
$$

where

$$
\zeta=\bigoplus_{p=0}^{n}(-1)^{p}: \bigoplus_{p=0}^{n} \Lambda^{p}[s, X] \mapsto \bigoplus_{p=0}^{n} \Lambda^{p}[s, X] .
$$

As $\zeta$ is a selfadjoint operator, we infer the formula

$$
\alpha((\tilde{a}, \tilde{b}))=v^{*}(\alpha(a) \otimes 1+\zeta \otimes \alpha(b)) v,
$$

which will be useful for our purpose. 
Let us return to the main idea of the proof. We have to show that

$$
\sigma(a, X) \times \sigma(b, Y) \subset \sigma((\tilde{a}, \tilde{b}), X \bar{\otimes} Y) .
$$

It will be sufficient to prove that if both $a$ and $b$ are singular systems then $(a, b)$ is itself singular.

(1) If there are $\xi \in \Lambda[s, X]$ and $\eta \in \Lambda[t, Y]$ with $\|\xi\|=\|\eta\|=1$ and $\alpha(a) \xi=0, \alpha(b) \eta=0$ then $\left\|v^{*}(\xi \otimes \eta)\right\|=1$ and by $(2.2) \alpha((\tilde{a}, \tilde{b})) v^{*}(\xi \otimes \eta)$ $=0$ hence $\alpha((\tilde{a}, \tilde{b}))$ is not invertible in this case.

(2) If there are $\xi \in \Lambda[s, X]$ and $\left\{\eta_{k}\right\}_{k} \subset \Lambda[t, Y]$ with $\|\xi\|=\left\|\eta_{k}\right\|=1$ and $\alpha(a) \xi=0, \alpha(b) \eta_{k} \rightarrow 0$ as $k \rightarrow \infty$ then $\left\|v^{*}\left(\xi \otimes \eta_{k}\right)\right\|=1$ and by (2.2) $\lim _{k \rightarrow \infty} \alpha((\tilde{a}, \tilde{b})) v^{*}\left(\xi \otimes \eta_{k}\right)=0$, hence $\alpha((\tilde{a}, \tilde{b}))$ is not invertible.

(3) If there are $\left\{\xi_{k}\right\}_{k} \subset \Lambda[s, X]$ and $\left\{\eta_{k}\right\}_{k} \subset \Lambda[t, Y]$ with $\left\|\xi_{k}\right\|=\left\|\eta_{k}\right\|=1$ and $\alpha(a) \xi_{k} \rightarrow 0, \alpha(b) \eta_{k} \rightarrow 0$ as $k \rightarrow \infty$ we have as above $\left\|v^{*}\left(\xi_{k} \otimes \eta_{k}\right)\right\|=1$ and $\lim _{k \rightarrow \infty} \alpha((\tilde{a}, \tilde{b})) v^{*}\left(\xi_{k} \otimes \eta_{k}\right)=0$ which shows again that $\alpha((\tilde{a}, \tilde{b}))$ is not invertible. Since both $\alpha(a)$ and $\alpha(b)$ are selfadjoint, it is clear that (1), (2) and (3) represent all the consequences of the singularity of $a$ and $b$ and in each case $\alpha((\tilde{a}, \tilde{b}))$ is not invertible.

2.3 Corollary. Suppose that $X_{1}, \ldots, X_{m}$ are nonnull complex Hilbert spaces and $a^{j}=\left(a_{1}^{j}, \ldots, a_{n_{j}}^{j}\right) \subset L\left(X_{j}\right)(j=1, \ldots, m)$ are commuting systems. If we define

$$
\tilde{a}_{k}^{j}=1 \otimes \cdots \otimes 1 \otimes a_{k}^{j} \otimes 1 \otimes \cdots \otimes 1 \in L(X),
$$

where $X=X_{1} \bar{\otimes} \ldots \bar{\otimes} X_{m}$, and $\tilde{a}^{j}=\left(\tilde{a}_{1}^{j}, \ldots, \tilde{a}_{n_{j}}^{j}\right) \quad\left(k=1, \ldots, n_{j} ; j=\right.$ $1, \ldots, m)$ then we have the equality

$$
\sigma\left(\left(\tilde{a}^{1}, \ldots, \tilde{a}^{m}\right) ; X\right)=\sigma\left(a^{1}, X_{1}\right) \times \cdots \times \sigma\left(a^{m}, X_{m}\right) .
$$

Proof. This corollary follows easily from Lemma 2.1 and Theorem 2.2 by an induction argument.

We obtain also from Theorem 2.2 some other consequences concerning the tensor product of operators, in particular the well-known result of Brown and Pearcy [1] (see [2] for details).

Thanks are due to D. Voiculescu for useful suggestions.

\section{REFERENCES}

1. A. Brown and C. Pearcy, Spectra of tensor products of opeators, Proc. Amer. Math. Soc. 17 (1966), 162-166.

2. Z. Ceausescu and F.-H. Vasilescu, Tensor products and Taylor's joint spectrum, Studia Math. (to appear).

3. A. T. Dash and M. Schechter, Tensor products and joint spectra, Israel J. Math. 8 (1970), 191-193.

4. S. Mac Lane, Homology, Springer, New York, 1970.

5. J. L. Taylor, A joint spectrum for several commuting operators, J. Functional Analysis 6 (1970), 172-191.

6. F.-H. Vasilescu, A characterization of the joint spectrum in Hilbert spaces, Rev. Roumaine Math. Pures Appl. 22 (1977), 1003-1010.

National Institute for Scientifical and Technical Creation, Bucharest, Romania 